\title{
NONLINEAR STABILITY ANALYSIS OF A GENERIC FAN WITH DISTORTED INFLOW USING PASSAGE-SPECTRAL METHOD
}

\author{
David Romera \\ School of Aeronautics and Space \\ Universidad Politécnica de Madrid \\ Madrid 28040, Spain \\ Email: david.romera@upm.es
}

\author{
Roque Corral* \\ Advanced Engineering Direction \\ Industria de TurboPropulsores S.A.U. \\ Madrid 28830, Spain \\ Email: roque.corral@itpaero.com
}

\begin{abstract}
The dependence of the aerodynamic stability of fan blades with the nodal diameter and amplitude of the inlet perturbations is studied. The analysis is conducted using a block-wise spatial Fourier decomposition of reduced-passages to reconstruct the full-annulus solution. The method represents very efficiently the unsteady flow generated by inlet non-uniformities. The explicit spatial Fourier approximation is exploited to characterize the relevance of each nodal diameter of the inlet perturbation in the fan stall process and study the nonlinear stability in a harmonic by harmonic basis. This approximation allows studying the contribution to stall of each circumferential mode separately. The methodology has been assessed for the NASA rotor 67 . The maximum amplitude of total pressure distortion at which the compressor becomes unstable and triggers a stall process has been mapped. It has been proven that despite the complexity of a screen-induced total pressure distortion the only relevant parameter for the nonlinear stability of the fan is the most unstable nodal diameter. The equivalence in terms of stability between realistic distortion screens and single harmonic distortions has been assessed. Full-annulus simulations have been conducted to assess the accuracy of the simplified nonlinear stability limit. It is concluded that performing a nonlinear simulation with the proper single harmonic perturbation is enough to assess fan stability. The total pressure error with respect the full annulus simulation including a screen-induced pressure deficit at the intake is
\end{abstract}

below $10 \%$. It is shown that for the NASA rotor 67 running at the nominal speed the most unstable nodal diameter is the first. This study not only shows a reduction in computational time to assess nonlinear fan stability by a factor of seven but also creates an efficient methodology for understanding the nonlinear instability of fans due to inlet distortion profiles.

\section{INTRODUCTION}

Aircraft designs wherein the propulsive turbomachines are partially or fully embedded within the airframe present serious challenges about the aerodynamic performance of the compression system. These problems are associated with the response to the distorted inflow caused by the nacelle and intake geometries, reducing the aerodynamic stability of the engine. Inlet distortion refers then to the nonuniform flow created upstream of the fan, or any other row of airfoils, which can detrimentally affect its performances in terms of efficiency or pressure rise. The performance and aerodynamic stability of fan blades operating in a circumferentially nonuniform inlet total pressure distribution was identified a long time ago as a key issue in the development of gas turbine engines and a serious concern in the design of turbofan engines [1]. Besides, to understand the effects of inlet distortion on the overall compression system and the engine, fan and compressor designs can be improved by specifically analyzing the influence of inlet distortion and how different patterns of total pressure distribution affect the stability of the compression system $[2,3]$. Non-uniform total pressure distribution at the inlet of the fan reduces its stable operating range and potentially 
creates forced response and mechanical integrity problems on the blades. This interaction and the effect of different inlet total pressure distributions become more important for the lip intake design of the new generation of short intake engines. Page et. al [4] calculated the stall inception process with different inlet distortions generated by curved intake ducts. The results provided evidence that the low momentum flow in the tip region leads to damaging instability. As a consequence, the relevance of studying fan stability under the effects of distorted inflow has been universally acknowledged.

The study of fan stability under the effect of specific profiles mimicking realistic environments, such as those caused by boundary layer ingestion $[5,6]$, flow separation in the inlet duct [7] or sudden manoeuvres of the aircraft [8], is an understudied part of the engine/airframe interaction and an active area of research [9]. Experimental and numerical investigation by $\mathrm{Hah}$ et al. [10] have shown that the inlet total pressure distortion increases aerodynamic losses when a strong unsteady interaction between the passage shock and the blade boundary layer develops. Further, experimental research on an $\$$-duct was completed by Ferrar et al. [11] and various mass flow rates tested. Results were similar to numerical simulations where curvature in the duct affects the static pressure gradient due to flow separation. The majority of recent works have focused on analyzing the effect on the stability of the distortion through a compressor stage. Zhang et. al [12] showed that for an inlet distortion with fixed strength, the stall margin loss increases as the rotational speed decreases, but very little attention has been paid to the effect of different circumferential extents and amplitude of the distortion pattern on the stability of the system.

A large number of experiments have been performed to explore the relationship between inlet distortion and compressor performance $[13,14,15,16]$. However, Computational Fluid Dynamics (CFD) provides an alternative method to determine the stability loss of a fan blade due to complicated inlet distortion patterns. Many researchers have assessed the effect of inlet distortion to understand, analyze, and predict its impact on the operation of an engine $[17,18,19,20,21]$. The common characteristic of these numerical strategies based on URANS simulations is their high computational cost. Modeling the aerodynamic effects using full-annulus transient simulations though feasible [22,23], is still too expensive for routinely used in design offices.

As an alternative to harmonic balance [24] or time-domain Fourier [25] reduced order methods, a time-marching PassageSpectral Method is proposed in this study to reduce the computational cost of these simulations and simplify the study of fanstage stability under the effect of distorted inflows without making any hypothesis about its temporal periodicity. An existing parallel CFD code $[26,27]$ has been modified and customized to study the aerodynamic response of turbomachinery rows due to distorted inlet flows by separating the contribution of each nodal diameter at the inlet. This approach, which has been verified against full-annulus cases for rotating stall and force response cases in an isolated fan-stage [28], has been further modified to deal efficiently with a single spatial harmonic and then applied to assess the effect of inlet distortion on the aerodynamic stability of the fan. In this model just three reduced-passages are retained. The understanding gained from this previous study with the current CFD code is used as a guideline for further numerical modeling in this work.

This piece of research proves that despite the complexity of screen-induced total pressure distortion, the most relevant characteristic to parameterize fan stability is the most nonlinearly unstable nodal diameter. In this work firstly, the nonlinear stability of each nodal diameter at the inlet is investigated as a function of the perturbation amplitude taking advantage of the use of the novel method. Next, the methodology is applied to a generic transonic fan blade with different total pressure distortion screens. Finally, an efficient methodology to assess fan stability on the presence of inlet distortion is proposed. In the authors opinion, this is the first time that clear conclusions are drawn on the influence of the inlet distortion shape on fan stability.

\section{NUMERICAL FORMULATION}

\section{Baseline Flow Solver}

The three-dimensional Navier-Stokes equations in conservative form for an arbitrary control volume can be written in compact form as:

$$
\frac{d}{d t} \int_{\Omega} \mathbf{U} d \Omega+\int_{\Sigma} \mathbf{F}(\mathbf{U}) \cdot d \mathbf{A}=0
$$

where $\mathbf{U}$ is the vector of conservative variables, $\mathbf{F}$ the sum of the inviscid and viscous fluxes, $\Omega$ the volume of the control volume, $\Sigma$ its boundary and $d \mathbf{A}$ the differential area pointing outward to the boundary.

The baseline solver, known as $M u^{2} s^{2} T$ [26], uses hybrid unstructured grids to discretize the spatial domain and may contain cells with an arbitrary number of faces. The governing equations 1 are discretized using a finite volume approach based on the dual mesh where the solution vector stores the variables at the vertexes of the mesh. The control volume associated with a node is formed by connecting the median dual of the cells surrounding it, using an edge-based data structure. The spatial discretization is formally second order accurate and a second-order backward implicit time integration is used. A matricial form of the numerical dissipation terms is used recurring to the Roe's matrix. The code is parallelized using MPI and executed in GPUs to reduce the turn around time [27].

Once the spatial domain is discretized, the preconditioned semidiscrete version of nonlinear equations 1 for the internal node $i$ 


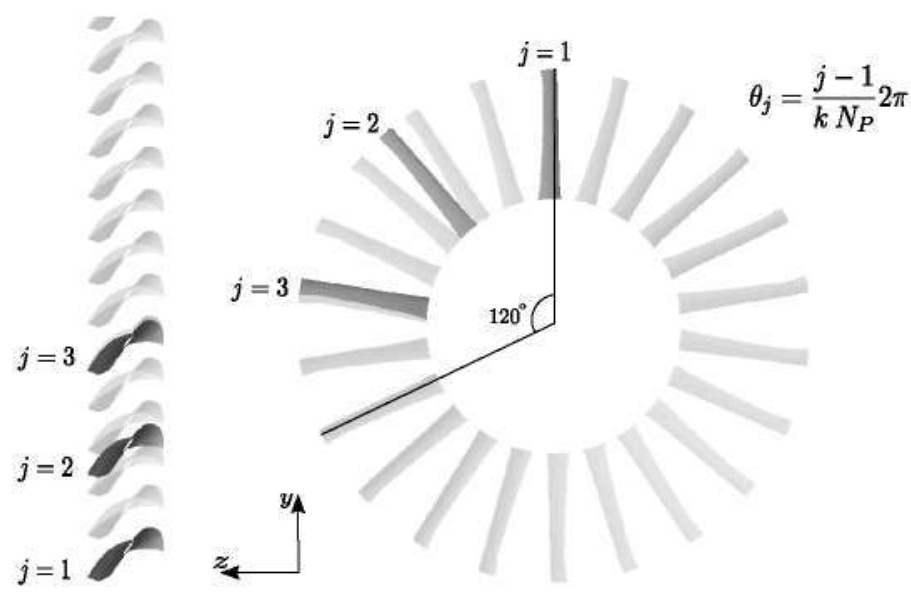

FIGURE 1. Sketch of the location of three-reduced passages to represent an inlet perturbation with $\mathrm{ND}=3$.

can be written as:

$$
\Omega_{i} \frac{d \mathbf{U}_{i}}{d t}+\sum_{j=1}^{n_{\text {edges }}}\left(\mathbf{F}_{i j}^{I+V} \cdot \sigma_{i j}-\mathbf{D}_{i j}\right)=\mathbf{S}\left(\mathbf{U}_{i}\right)
$$

where $\Omega_{i}$ is the control volume, $\sigma_{i j}$ is the area associated with the edge $i j, \mathbf{F}_{i j}^{I+V}$ represents the inviscid and viscous fluxes through this area, $\mathbf{D}_{i j}$ are the artificial dissipation fluxes, $\mathbf{S}$, represents the source terms, and $n_{\text {edges }}$ the number of edges surrounding node $i$. Turbulence effects are accounted for using the $k-\omega$ model with realizability, which is integrated to the wall, or the Baldwin and Lomax algebraic eddy-viscosity model. Equation 2 can be written in compact form as:

$$
\frac{d \mathbf{U}_{i}}{d t}=\mathbf{R}(\mathbf{U})
$$

The vectors of unknowns, $\mathbf{U}$, and residuals, $\mathbf{R}$, in a full-annulus simulation can be split in a passage basis as

$$
\mathbf{U}=\left(\begin{array}{c}
\mathbf{U}_{i}^{1} \\
\mathbf{U}_{i}^{2} \\
\cdot \\
\cdot \\
\cdot \\
\mathbf{U}_{i}^{N_{b}}
\end{array}\right), \mathbf{R}=\left(\begin{array}{c}
\mathbf{R}_{i}^{1} \\
\mathbf{R}_{i}^{2} \\
\cdot \\
\cdot \\
\cdot \\
\mathbf{R}_{i}^{N_{b}}
\end{array}\right)
$$

where the column vector $\mathbf{U}^{j}$, contains just the unknowns of the $j-t h$ passage, $N_{b}$ is the number of rotor blades or vanes of the row, and the node $i$ represents homologous nodes in different passages.

\section{Block Spectral Method}

The proposed method has been previously described in [28] and the reader is referred to this reference for further details. This method has been modified and customized for this work to study the contribution of a given single spatial harmonic $k$ efficiently, by approximating the solution with only three reduced-passages samples spanning over a sector of size $2 \pi / k$.

The code is based on the virtual existence of a Fourier transformation of the homologous nodes of the different passages, $\mathbf{U}_{i}^{j}$, and the description of the flow in terms its Fourier coefficients:

$$
\hat{\mathbf{U}}_{i}^{m}(\mathbf{x}, t)=\sum_{j=1}^{N_{p}-1} \mathbf{U}_{i}\left(\mathbf{x}, \theta_{j}, t\right) e^{-i m \theta_{j}}
$$

where

$$
\theta_{j}=\frac{j-1}{k N_{p}} 2 \pi
$$

The purpose of using a Fourier modeling of the spatial flowfield is to use a truncated Fourier series of the "exact" solution and retain only the most representative harmonics. The underlying hypothesis is that the wavelength of the circumferential non-uniformities is long, and therefore, a few harmonics suffice to represent the long wave-lengths. Short wave-length variations are retained within the blade passage mesh. Obviously, the Fourier transform of all the mesh nodes is impractical and does not need to be computed. Since the numerical scheme is local in nature, only the nodes located near the periodic boundaries need to be informed and corrected with the global information carried by the Fourier coefficients. In this fashion, only a small subset of nodes need to be Fourier transformed.

If only a single arbitrary harmonic of the perturbed flow is retained then just three equally spaced passages need to be computed to reconstruct the mean value and the harmonic. The reason to choose three reduced-passages samples to solve the first spatial harmonic is because to approximate a sinusoidal function using a discrete space Fourier series you need to calculate three unknown variables: the mean value, $\bar{U}$, and two Fourier coefficients, $A_{k}$ and $B_{k}$, representing the $\sin (k \theta)$ and $\cos (k \theta)$. This turns into a system of three equations with three unknown variables. This system is solved for each of the new neighboring nodes. The main idea of the modified method is sketched in Fig. 1 where it can be seen that the third harmonic $(k=3)$ of the circumferential variations of the flow-field is reconstructed using only three passages (in dark gray) in a $120^{\circ}$ sector to approximate the flow due to an excitation containing just the third nodal diameter. The solution in the rest of the passages (in light gray), inside and outside of the $120^{\circ}$ sector, is interpolated using a Fourier reconstruction using solely the information of the three single-passage domains and the periodicity condition in the studied $120^{\circ}$ sector.

\section{FAN STABILITY}

This section aims at studying the nonlinear aerodynamic stability of a generic fan blade operating at a constant speed under distorted inflow. Intake distortion is parameterized in terms of 


\begin{tabular}{|l|c|}
\hline Number of rotor blades & 22 \\
\hline \hline Nominal Rotational speed $[\mathrm{rpm}]$ & 16043 \\
\hline Mass-flow rate $[\mathrm{kg} / \mathrm{s}]$ & 35.25 \\
\hline Pressure ratio & 1.56 \\
\hline Tip-clearance $[\mathrm{mm}]$ & 0.61 \\
\hline
\end{tabular}

TABLE 1. NASA rotor 67 design operating conditions.

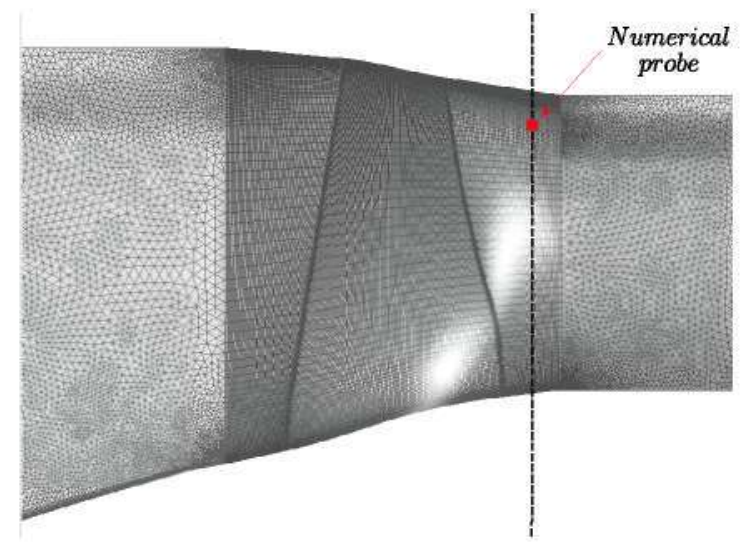

FIGURE 2. NASA rotor 67 semi-unstructured grid.
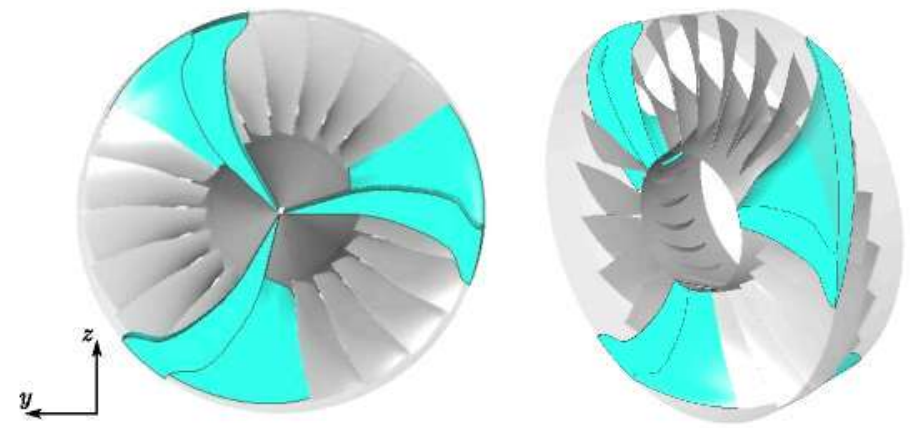

FIGURE 3. NASA rotor 67 full computational domain (3 reducedpassages) for a $N D=1$ approximation.

its nodal diameter and amplitude. The idea of characterizing the effect of inlet distortion on fan stability using the Fourier coefficients of the inlet perturbation was pursued by Peterson et al. [29], but their studies were inconclusive. Here this same idea has been seeked but more systematically and with different numerical tools.

The non-uniformity of the incoming flow gives rise to nonuniform circumferential total pressure distortions which in turn create a strong aeromechanical response in the rotor blades, compromising fan stability [7]. The incoming and the result- ing flow disturbances are usually dominated by long circumferential wave-lengths (i.e.: much longer than the blade pitch) which makes the Passage-Spectral Method (PSM) well suited for its analysis since it avoids the use of full-annulus simulations. Moreover, the method allows investigating the independent contribution of the different NDs efficiently. It is important to point out that this is not the case in nonlinear full-annulus simulations since when the perturbations are large, and the linear stability regime is by-passed, the nonlinear interaction amongst the different NDs is unavoidable. However, the PSM method intrinsically works in a nodal diameter basis and the modal content of the solution can be tailored and filtered out. In other words, the PSM naturally allows to investigate the contribution to the nonlinear stability of the system of each spatial circumferential mode, whereas full wheel analyses not since the flow non linearity can gives rise to complex nonlinear interactions.

In this regard, the method has been adapted to simulate an arbitrary single ND perturbation using solely three reduced passages to fit the desired wave-length. This implies that, by construction, the nonlinear interaction amongst different NDs is excluded. The method allows to study the contribution to the stability of the different spatial harmonics separately, just retaining three reducedpassages over a sector of $2 \pi / N D$ degrees (see Fig. 1), and simulate the effect of this single harmonic filtering the nonlinear contribution of the rest. Two types of simulations are used in this work. The first are full-annulus simulations which are used as a high-order model for verification purposes. The second are PSM simulations with a single spatial harmonic or ND matching that of the inlet perturbation. This approach is very efficient and is used to map the design space.

The NASA rotor 67 , which is a low-aspect-ratio fan, has been chosen as the test vehicle. Design parameters of the fan are listed in Table 1 at $\% 100$ of the design speed and a view of the fan semistructured grid [26] is given in Fig. 2. Firstly a hybrid grid in the blade-to-blade plane with 118 points around the airfoil and 24 quad layers in the boundary layer has been constructed, next 151 layers in the span-wise direction are extruded. To resolve the tip leakage flow 6 mesh layers were included between the blade tip and the casing. The final grid consists of about $2.27 \times 10^{6}$ nodes per passage. The size of the full-annulus mesh is about $50 \times 10^{6}$ nodes. A duct of approximately 2 rotor chords has been added upstream and downstream of the rotor to ease fan stability analysis although it is acknowledged that the stability margin depends on this location [8].

One-dimensional non-reflecting boundary conditions were imposed at the inlet and the outlet. At the exit, the radial equilibrium equation was applied to fix the pressure radial distribution. The Reynolds number of the simulations is very high $\left(\operatorname{Re} \sim 10^{6}\right)$ and the standard $k-\omega$ turbulence model with wall functions was used to alleviate mesh requirements in the boundary layers. The goal is to use three reduced-passages to capture the large-scale 


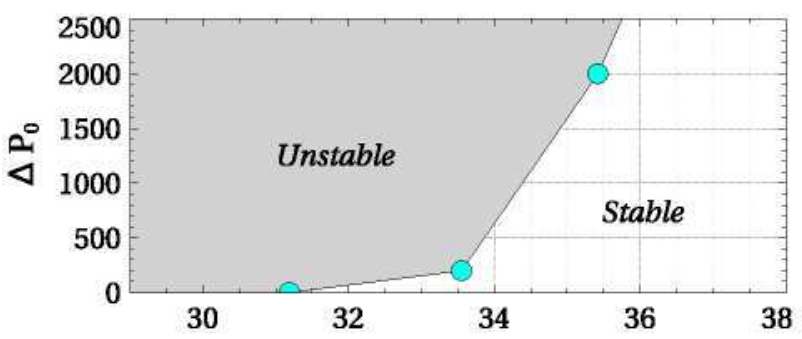

(a)

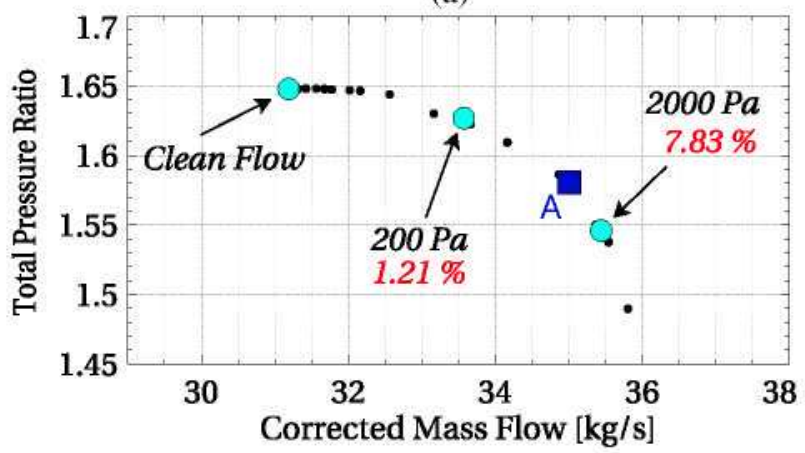

(b)

FIGURE 4. Effect of inlet distortion amplitude at $100 \%$ of the design speed for $N D=1$ computed with the PSM.

nonaxisymmetric perturbations of the flow and predict the trigger of the stall process under distorted inflow, filtering the harmonics which are different than the nodal diameter at the inlet. Figure 3 displays the computational domain and the three reduced-passages required to carry out the spatial Fourier transformation for a single spatial harmonic and the whole length of the computational domain.

Unsteady simulations have been conducted using 400 time steps per rotor revolution and run for 22 blade passing periods. The number of pseudo time steps per physical time step was fixed to 60 , which is large enough to achieve the convergence criterion of the inner iteration of the dual time step. The base flow is uniform and axial at the inlet, but the total pressure is modified circumferentially by superimposing an arbitrary Fourier series but keeping a uniform radial distribution. Using this technique either simple sinusoidal perturbations in the azimuthal direction or standard $60^{\circ}$ or $90^{\circ}$ inlet distortion screens can be created.

\section{Nonlinear Stability}

The effect of varying the amplitude of the inlet stagnation pressure first nodal diameter pattern on the fan stability at $\Omega_{\text {rotor }} / \Omega_{\text {nominal }}=100 \%$ is investigated first with the present method. In this case, the $P_{0}$ at the inlet can be expressed as $P_{0}=\bar{P}_{0}+\Delta P_{0} \sin \theta$. Figures $4(a)$ and (b) show the stall margin as a function of the corrected mass flow. The blue circle with the minimum mass-flow coincides with the last converged point with the single-passage steady version of the solver. At this OP the fan is unstable under infinitely small perturbations. As the mass-flow is increased the fan is more tolerant to inlet perturbations. This process is sketched in Fig. 4. At the bottom the blue circles represent the mean operating point with the inlet perturbation included. This means that these points are the result of a time averaging procedure of the unsteady solutions. It can be noticed that the deviation with respect the speedline computed using single-passage steady simulations is small.

The stall margin loss can be defined as the deficit of stall margin with inlet distortion

$$
S M_{L}=\frac{P R_{\text {ref }}-P R_{s}}{P R_{\text {ref }}} \times 100 \%
$$

where $P R_{\text {ref }}$ and $P R_{s}$ are the total pressure ratio of the near stall point with clean flow and distorted inflow, respectively. It can be seen that as the amplitude of the 1st ND perturbation becomes higher, from an amplitude of $200 \mathrm{~Pa}$ in Fig. 4(b) to $2000 \mathrm{~Pa}$, the stall margin loss of the blade raises from $1.21 \%$ to $7.83 \%$. The stall margin loss increases steadily with the distortion amplitude. This behavior is usually explained by noting that for large total pressure perturbations the airfoils operate during part of the period in off-design conditions making some of the blades more prone to separation triggering the whole fan in stall.

Figure 4(a) shows the nonlinear stability map for the 1st ND computed with the PSM. This case exhibits a subcritical bifurcation, i.e.: OPs which are stable under small perturbations can become unstable in the presence of large disturbances. This instability process is nonlinear and for small perturbations this phenomenon cannot be seen. Its simulation requires of fully nonlinear analysis. Figures 4 (a) and (b) show that the farther apart the $\mathrm{OP}$ from the stall line the larger the disturbance required to drive the fan into a massive stall. Large perturbations can trigger an intrinsically nonlinear stall process. This behavior is well known and has been reported and simulated by several authors $[2,4,8,12,16]$.

Traditionally, fan stability has been experimentally and numerically investigated using inlet distortion screens to perturb the incoming flow. These perturbations are physically implemented partially blocking a sector of the intake. Here, the difference is that taking advantage of the PSM the contribution of the different NDs can be investigated independently preventing the interaction between them. The stability of the fan has been investigated in a nodal diameter basis retaining the spatial harmonic corresponding to the inlet perturbation solely.

Figure 5 depicts the nonlinear stability map of the first four NDs at the inlet. The unstable regions are shaded in color. It can be seen that the contribution of the different NDs to the nonlinear stability is qualitatively different. The $\mathrm{ND}=1$ has a sub-critical bifurcation while that of the ND=3 is supercritical. However, the stability of the NDs two and four is practically not altered by the level of the perturbation. The four nearly straight lines depart 

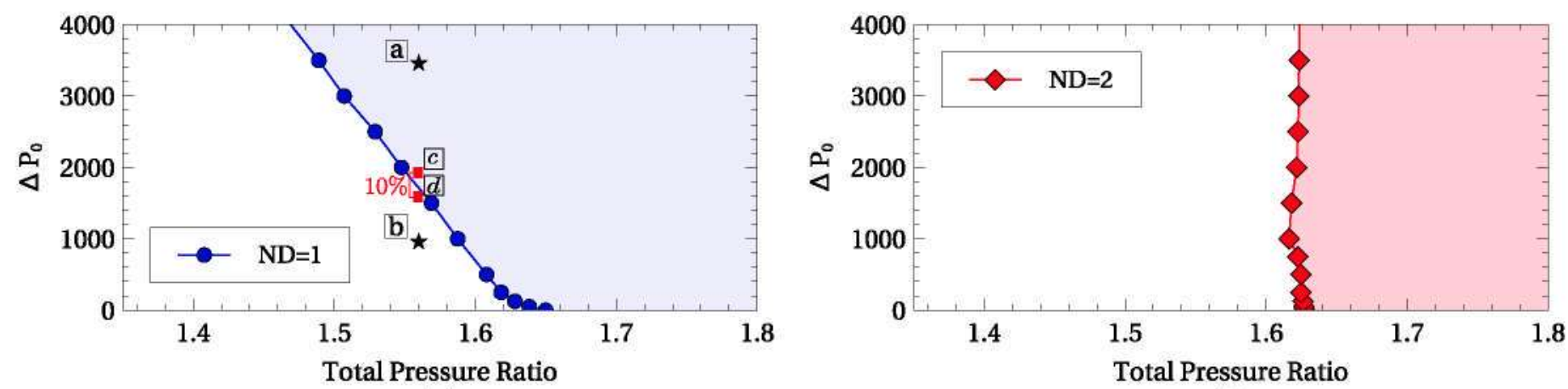

(a)
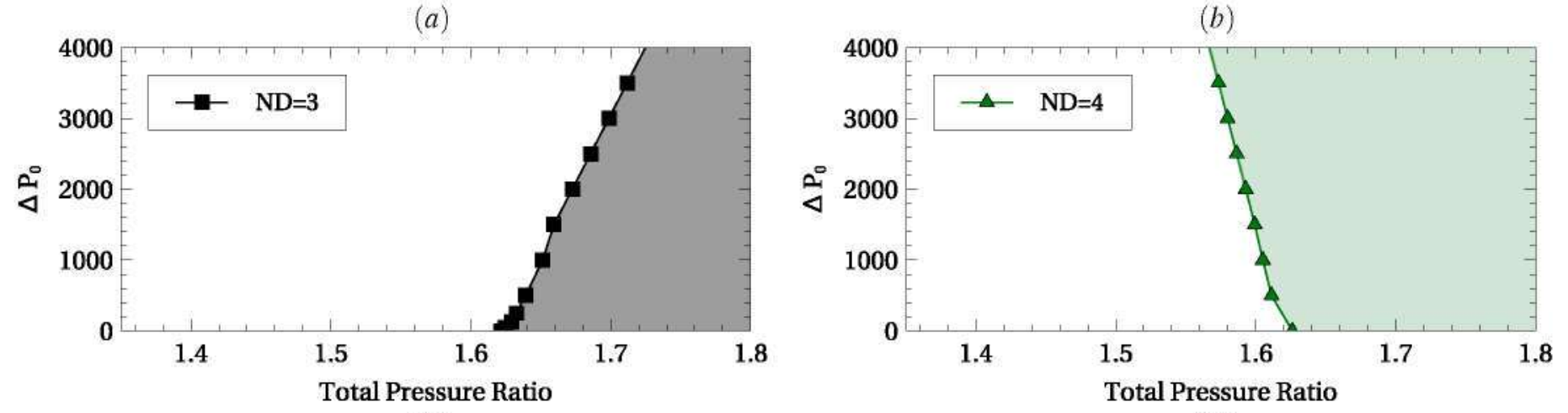

(c)

(d)

FIGURE 5. Stability conceptual map due to different amplitudes of inlet distortion distribution at $100 \%$ of the design speed and nodal diameters, $N D$, using the proposed PSM just retaining the harmonic $k=N D$.

from the same point, at least with the error of the method, which is the linear or small perturbation stability limit. The first ND has the largest sensitivity to inlet perturbations, and in practice, its behavior is the one observed with realistic, non-sinusoidal, perturbations. The most surprising result of Fig. 5 is the stabilizing behavior of $\mathrm{ND}=3$. The actual interpretation of Fig. $5(\mathrm{c})$ is that the fan could operate beyond the linear stability limit if the sole forcing at the inlet corresponds to the ND three, and this is high enough. In this case, the fan exhibits a periodic but stable response to this type of forcing. The combination of ND three with other NDs, such as the first, has not been further pursued.

The effect of the 1st ND inlet total pressure amplitude on fan stability has also been investigated at $\Omega_{\text {rotor }} / \Omega_{\text {nominal }}=50 \%$. It is concluded that, in this case, the stability of the fan depends very weakly on the perturbation amplitude for all the NDs and therefore the overall stability of the fan coincides with the obtained for the small perturbation regime. This means that the contribution of the inlet distortion to the change in the fan stall margin of different total pressure patterns depends on the rotational speed [12].

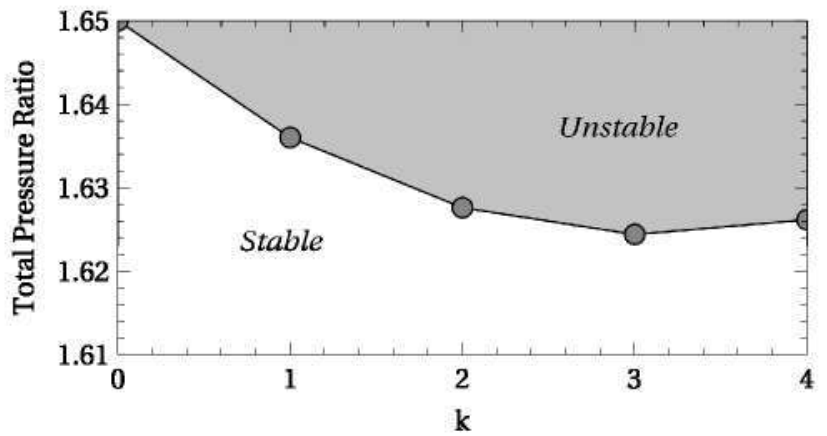

FIGURE 6. Stability conceptual map with clean inflow using PSM with a single harmonic $k$ at $100 \%$ of the design speed.

\section{Linear Stability}

The stability of the fan-stage is now studied for clean inflow conditions. The simulations are set with a constant uniform total pressure at the inlet and a single harmonic, $k$, of the PSM is computed. This is physically equivalent to compute different sector sizes. For instance if $k=3$ the problem would equivalent to run a $120^{\circ}$ sector and filter out all the contributions corresponding to the rest of NDs. The pressure ratio is modified by changing the level of the circumferentially uniform exit static pressure. 
Figure 6 shows the linear stability threshold as a function of the spatial harmonic index or sector size. It can be seen that the most unstable ND is the third, but the difference between different NDs is not high, The zero nodal diameter is the most stable. This means that if single-passage simulations were run to drive the code to stall at constant speed, before this actually happened, the equivalent full-annulus simulation would transition to an unsteady rotating stall pattern. The points of Fig. 6 represent the crossing of the stability limit of the different NDs of Fig. 5 with the horizontal axis. The departing pressure ratio changes slightly with the ND, exactly as shown in Fig. 6.

This type of plot is difficult to construct using full-annulus simulations, especially if the stabiliy limit of the different modes is close, since it is not possible to control the development of all the unstable NDs when they are active simultaneously in a single simulation. According to Fig. 6 , the fan cannot operate in a stable manner for pressure ratios higher than 1.65. However, Fig. 5(c) suggests that if a high enough perturbation with the third nodal diameter pattern is introduced the fan could operate in a stable manner beyond this point, which is an interesting conclusion of this study due to its potential application.

Finally, it is important to highlight that while the most unstable nodal diameter in a linear sense is $\mathrm{ND}=3$, the most critical ND is the first, and the one which can be triggered by a finite-amplitude perturbation in the linearly stable region. This means that linear fan stability analysis is misleading in most of the practical situations.

\section{RESULTS}

The goal of this work is to prove that though screen-induced distortion is complex since involves many Fourier spatial modes, the only relevant feature to characterize fan nonlinear stability is the most nonlinearly unstable nodal diameter. Although in the context of linear stability this statement is obvious, in the present problem which involves highly nonlinear mechanisms this statement needs to be proven. To do that a numerical strategy has been developed to study the nonlinear stability of a fan-stage under the effect of different total pressure distortion screens in the stationary frame of reference by approximating it using Fourier series and analyzing the stability of each of the harmonics in a separate way.

A physically induced irregularity in the approaching flow can lead to a premature dynamic separation of the blades propagating along with the compressor circumferential direction in the sense of rotor rotation. This instability destroys the cyclic symmetry of the flow since typically a fraction of the wheel contains stalled airfoils while the remaining exhibits attached flow. The sinusoidal shape of the inlet distortion profile promotes the development of transient low-order spatial harmonics. This is in contrast to other types of inlet distortion where a stepped total

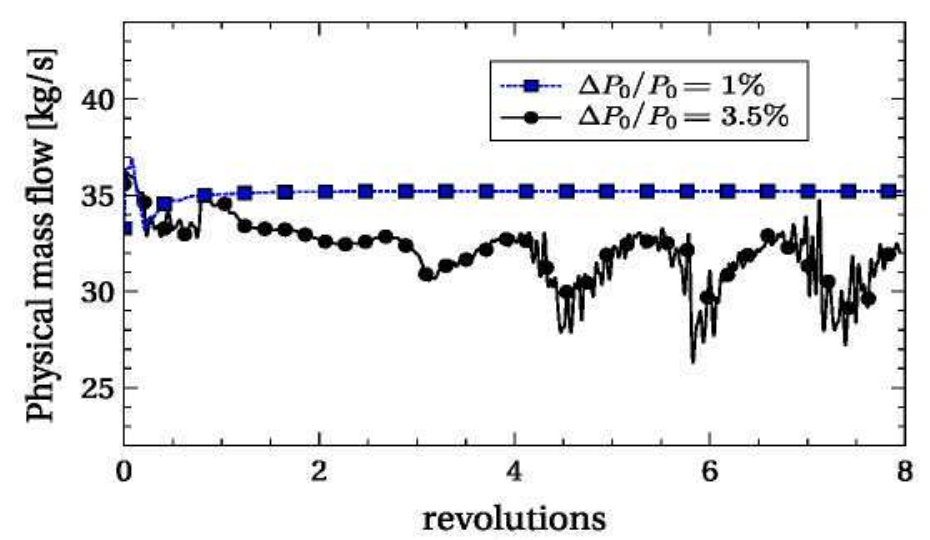

(a)

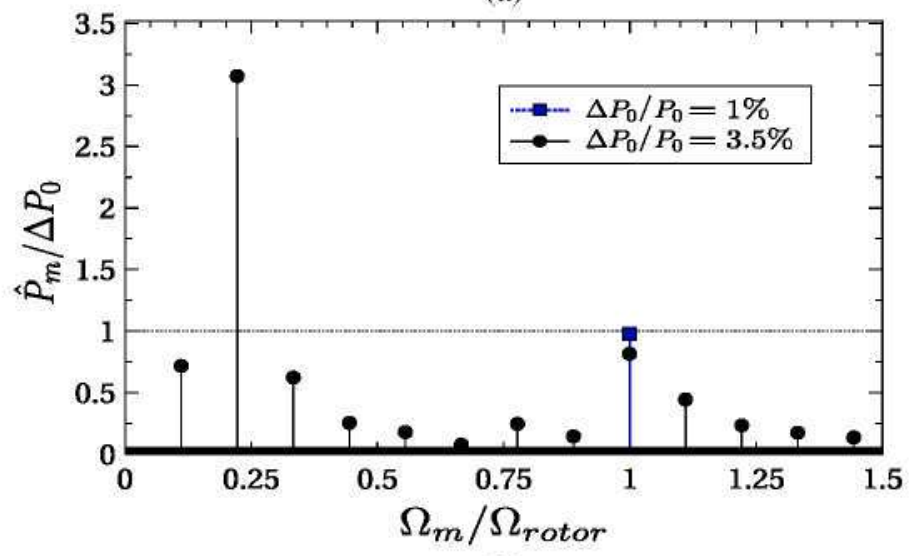

(b)

FIGURE 7. Full-annulus comparison between stable and unstable conditions with a first nodal diameter $(N D=1)$ perturbation with amplitudes of $\triangle P_{0}=1000 \mathrm{~Pa}$ and $\triangle P_{0}=3500 \mathrm{~Pa}$, marked as a and b in Fig, 5(a) respectively, for the 1st harmonic component $(m=1)$ of the total pressure signal at 0.5 axial chords downstream of fan. $P_{0}=101325 P a$.

pressure distortion screen is used to represent the blockage at the inlet [8]. However, as we will show in the following sections, both inlet distortion patterns can behave similarly.

The OP marked as point A ( $\square$ ) in Fig. 4(b) was chosen to investigate this point. The steady solution at $100 \%$ of the design speed with uniform inlet stagnation pressure at a near peak efficiency operating point is used as the initial solution for the unsteady simulations.

\section{Stability Map Validation}

The full-annulus solutions obtained for the points marked as a and $b$ in Fig. 5(a), and which correspond with two different perturbation levels of the 1st ND, are described next. Figure 7(a) compares the the mass-flow time evolution during the first 8 revolutions at the rotor outlet for two levels of perturbation, 
$\triangle P_{0} / P_{0}=1 \%$ and $3.5 \%$ which correspond to stable and unstable full-annulus cases respectively. For the stable case, it can be seen how following the initial perturbation the mass-flow converge to a stable solution, though the simulation is fully unsteady, whereas for the unstable case the mass-flow drops by about $15 \%$ and has an erratic behavior.

Figure 7(b) displays the frequency spectrum in the relative frame of reference of the total pressure signal in a numerical probe located near the outer casing 0.5 axial chords downstream of the fan (see Fig. 2). The horizontal axis of Fig. 7(b) represents the angular velocity of propagation of the spatial harmonic $m$ relative to the rotor, $\Omega_{m}=\omega_{m} / m$, non-dimensionalized with the rotor speed, $\Omega_{\text {rotor }}$. The spectrum of the stable case with $\triangle P_{0} / P_{0}=1 \%$ shows a single perturbation propagating with the rotor speed caused by the total pressure distortion at the inlet. The amplitude of this perturbation nearly coincides with that of the inlet meaning that its attenuation is very low. This case reduces to a pure forced response case with a single fundamental frequency.

On the contrary, the spectrum of the unstable case $\left(\triangle P_{0} / P_{0}=\right.$ $3.5 \%$ ) is rich in frequencies due to the stall process. The fundamental frequency created by the inlet perturbation is also seen. Additionally, the propagation velocity of the stall cell which is about $25 \%$ of the rotor speed (in the relative frame of reference) is clearly distinguished. The unsteadiness created by the stall cell is nearly three times bigger than that created by the sinusoidal perturbation at the inlet.

\section{Equivalent Inlet Distortion Principle}

Complex inlet distortion patterns can be Fourier transformed and then the nonlinear stability maps shown in Fig. 5 used to assess the fan stability in a nodal-diameter by nodal-diameter basis. As it has been mentioned before, it is unclear why this idea should work at all in a strongly nonlinear problem when several NDs of finite amplitude are superimposed.

With the objective in mind of proving this hypothesis, two inlet distortions patterns with $120^{\circ}$ and $60^{\circ}$ inlet screens were created and simulated using full-annulus analyses. The total pressure defect in the $120^{\circ}$ and $60^{\circ}$ inlet screens were chosen to be approximately $15 \%$ and $25 \%$ of the inlet total pressure respectively, leading in both cases to the same amplitude of the first harmonic, namely $\triangle P_{0} \simeq 3500 \mathrm{~Pa}$. This case corresponds to point $\mathrm{a}$ in Fig. 5 (a) which according to the proposed methodology should be unstable. The two different virtual inlet screens and their Fourier content can be seen in Fig. 8. These simulations are compared with a third case consisting of a simple 1st ND sinusoidal pattern at the inlet with the same amplitude, $\triangle P_{0} \simeq 3500 \mathrm{~Pa}$. It can be verified using Fig. 5, that the only unstable mode of the harmonic content of both screens is the 1st ND.

The hypothesis of the proposed approach has been confirmed us-

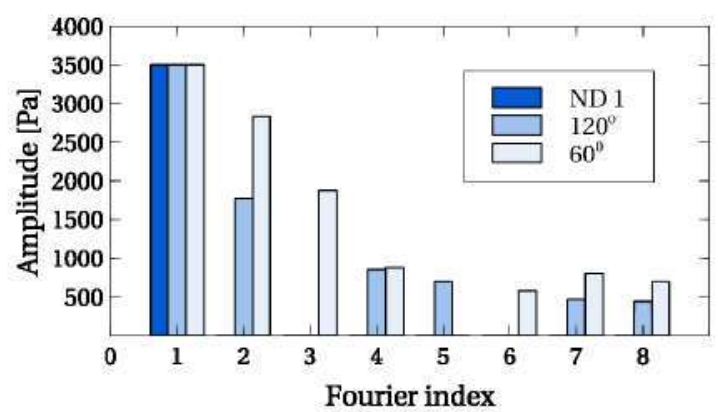

(a)

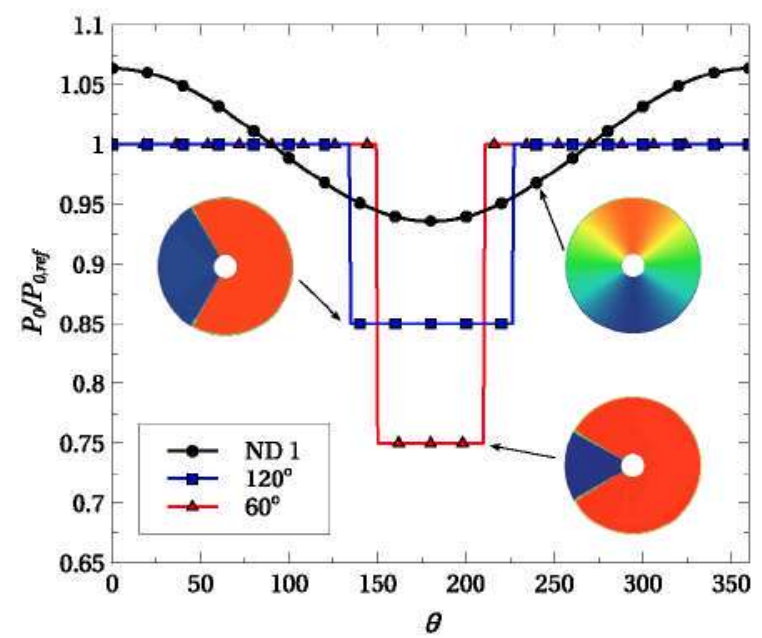

(b)

FIGURE 8. Fourier approximation (top) and total pressure inlet distortion profile (bottom) for the $120^{\circ}$ and $60^{\circ}$ screens with $15 \%$ and $25 \%$, respectively of inlet total pressure deficit, and the equivalent sinusoidal perturbation. $\left(P_{0, \text { ref }}=101325 \mathrm{~Pa}\right)$.

ing full annulus simulations. From a stability point of view, the points $\mathrm{a}$ and $\mathrm{b}\left(\triangle P_{0} \simeq 1000 \mathrm{~Pa}\right.$. $)$ in Fig. 5 (a) computed using a $60^{\circ}$-sector, a $120^{\circ}$-sector and a sinusoidal perturbation lead to the same conclusion: Point $a$ is unstable and Point $b$ is stable. This is consistent with the location of both points with respect to the neutral line. Though the answer is correct, it could be argued that points $a$ and $b$ are located far away from the neutral line, and therefore this approach is only qualitatively correct.

To assess the accuracy of the stability criterion based on the analysis of the individual spatial Fourier modes, the nonlinear stability threshold for the $60^{\circ}$ inlet distortion screen has been found resorting to full-annulus simulations. Departing from point $a$, the total pressure defect of the screen has been gradually decreased until approximately the stability limit obtained using the PSM which is $\Delta P_{0, c} \simeq 1800 \mathrm{~Pa}$, corresponding to a total pressure defect of the screen of approximately $15 \%$. The inlet background total pressure is kept constant during the whole iterative process. 
An equivalent process was followed for point $\mathrm{b}$. Points $\mathrm{c}$ and d are the results of this process, as shown in Fig. 5(a), and bracket the stability limit for the amplitude of the 1st harmonic between $\Delta P_{0, c} \simeq 1800(1 \pm 0.1) \mathrm{Pa}$. The iterative process based on a series of full-annulus simulations was stopped when the error in the perturbation was $10 \%$, with respect to the threshold provided by the simplified PSM method. It can be stated that point $d$ is stable whereas $\mathrm{c}$ is not. Further points, closer to the stability limit delivered by the PSM method, were not attempted.

\section{Statistically Converged Flow Description}

Figures 9(a), (b) and (c) show the instantaneous contours of the relative total pressure 0.5 axial chords downstream of the fan after 8 rotor revolutions for the point a in Fig. 5 (a) $\left(\Delta P_{0}=3,500 \mathrm{~Pa}\right.$ and $\mathrm{PR}=1.56$ ). When the flow is destabilized a stall cell is created in a low total pressure region near the tip of the blade created by the inlet distortion. The separation begins to grow in this low-pressure region and the stall is triggered. Once the perturbation created in the inlet impinges the rotor, two more revolutions are needed to form a fully developed stall cell. It was shown, both experimentally [16] and numerically [8], that the flow disturbances with frequencies different from the fundamental one associated with the inlet perturbation trigger the stalling of the blade. Such frequencies cannot be modeled with single-passage method using phase-shifted boundary conditions since the basic assumption is that there is a unique and known fundamental frequency. This is the underlying reason why fullannulus solutions have been used for these complex cases until now. However, the PSM, even with a single spatial harmonic, can reproduce the frequency spectrum quite decently with the corresponding reduction in computational resources. It has been previously demonstrated as well that the present method can reproduce fan transients caused by inlet distortion with a low number of spatial harmonics [28].

To further study this statistically converged quasi-periodic state the time-varying amplitude of the spatial harmonics has been Fourier transformed in time. First, the spatial Fourier transform of the selected points is performed and then the Fourier coefficients are Fourier transformed in time again. The tracking point where the time-varying total pressure signal is studied, is located in the rotor frame of reference near the casing 0.5 axial chords downstream of the fan trailing-edge. It was found that the flow takes about 8 rotor revolutions to reach a converged quasi-periodic state. To obtain a clean signal the initial part of the transient was discarded in the analysis. The dimensionless phase velocity spectra are presented in Figs. 9(d), (e) and (f) where the horizontal axis represents the angular velocity of propagation of the harmonic relative to the rotor, $\Omega_{m}=\omega_{m} / m$, nondimensionalized with the rotor speed, $\Omega_{\text {rofor }}$. It can be seen that the spectrum is rich in frequencies as a consequence of the interaction of the stalled cells with inlet non-uniformity.

Synchronous Response The synchronous response of all the fan harmonics due to the inlet non-uniformity travels with the rotor velocity in the relative frame of reference and can be distinguished in Figs. 9(d), (e) and (f) at $\Omega_{m} / \Omega_{\text {rotor }}=1$. It is important to recall at this point that Fig. 9 (bottom line) displays the spatial mode amplitude as a function of the phase speed $\Omega_{m}=\omega / \mathrm{m}$. and therefore all the incoming are superimposed at $\Omega_{m} / \Omega_{\text {rofor }}=1$. The amplitude of the first harmonic, $m=1$, is the same for the three cases and approximately equal to $\triangle P_{0} \simeq 3500 \mathrm{~Pa}$. Figures $9(\mathrm{e})$ and (f) which correspond to the $120^{\circ}$ and $60^{\circ}$ screens respectively, show contributions as well from higher order modes. The mode $m=2$ appears both in the $120^{\circ}$ and $60^{\circ}$ cases. However, this is not the case for the mode $m=3$, whose contribution is zero for the $120^{\circ}$ case. This is consistent with the frequency content of the incoming perturbation, see Figs. 8(c) and (d). It is important to note the third harmonic of the $120^{\circ}$-screen is zero. This is why it does not show up at at $\Omega_{m} / \Omega_{\text {rotor }}=1$ in Fig. 9(e). Though this conclusion is obvious, it is important to remind it for a correct interpretation of Fig. 9.

Asynchronous Response The patterns traveling at a speed different from the angular rotor velocity appearing in Figs. 9(d), (e) and (f), correspond to that generated by the stall process. When the inlet perturbation impinges the rotor, low frequencies whose main spatial content corresponds to the 1st ND $(m=1)$ consistently appear, see Figs. 9(d), (e) and (f). The propagation velocity of this pattern is about $25 \%$ of the rotor velocity in the relative frame of reference in all the three cases. A single stall region is created in all three cases. It is important to highlight that the 1st ND diameter was identified by the nonlinear stability analysis as the most promising candidate to be triggered by a finite amplitude excitation.

The tip leakage vortex shows a periodic behavior as the rotor enters and exits the distorted region. Rotating stall will happen when the flow separation generated in the distorted region is not removed in the clean flow area [8]. These disturbances are almost constant along time, reaching a quasi-steady state. After about 3 rotor revolutions (see Fig. 7), the mass flow decreases to stall levels and the low-frequency content of the spectrum increases, what is a natural consequence of stall process.

The first ND has the largest contribution to the stall cell and its unsteadiness level is three times bigger than that created by the inlet non-uniformity. The spectrum has a complex harmonic content but in overall they are alike. Obviously, the spatial content of the simulation performed with the PSM method using a single spatial harmonic has fewer spatial scales but the frequency content is similar. The method can reproduce the one stall rotating 


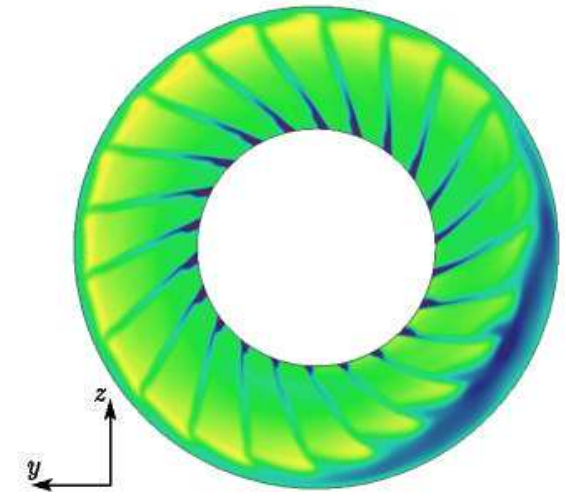

(a) 1st ND sinusoidal screen (PSM $k=1)$

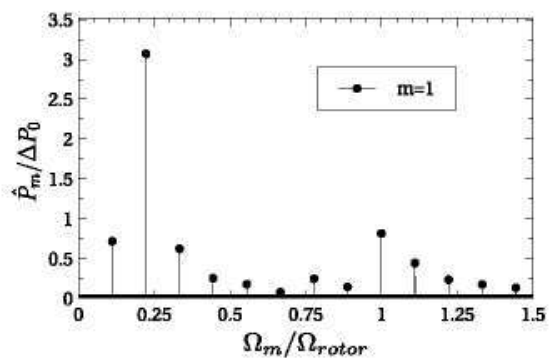

(d) 1st ND sinusoidal screen (PSM $k=1$ )

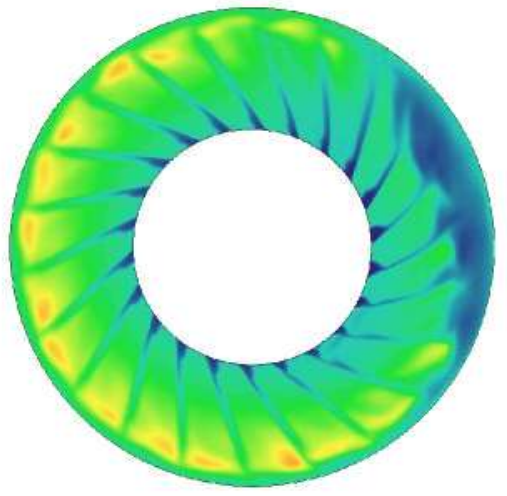

(b) $120^{\circ}$ screen (Full-annulus)

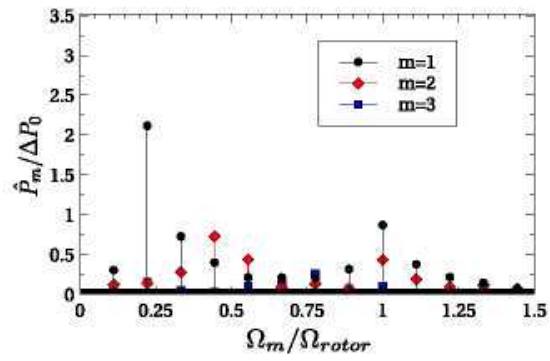

(e) $120^{\circ}$ screen (Full-annulus)

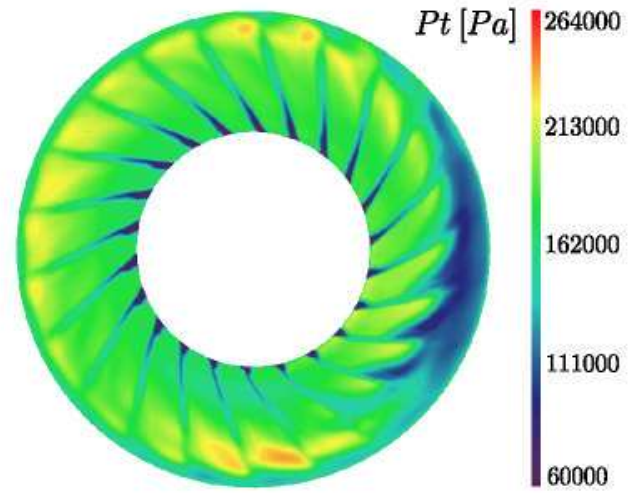

(c) $60^{\circ}$ screen (Full-annulus)

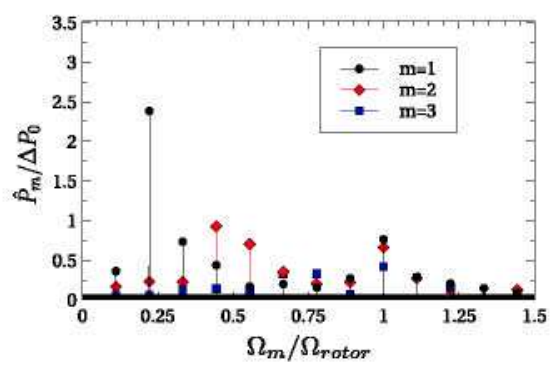

(f) $60^{\circ}$ screen (Full-annulus)

FIGURE 9. Comparison for the three different inlet distortion screens at $100 \%$ of the desgin speed, (a) and (d): sinusoidal screen, (b) and (e): $120^{\circ}$ screen, (c) and (f): $60^{\circ}$-screen. Top: Snapshoot of the relative total pressure contours 0.5 axial chords downstream of the fan after 8 rotor revolutions non-dimensionalized with the inlet total pressure amplitude of the 1st spatial harmonic, $\triangle P_{0}$. Bottom: non-dimensional relative angular phase velocity of the spatial harmonics, $\Omega_{m} / \Omega_{\text {rotor }}$, for the three inlet distortion screens of a total pressure probe near the outer casing 0.5 axial chords downstream of the fan (see Fig. 2) non-dimensionalized with $\triangle P_{0}$.

cell of the full-annulus solution with reasonable accuracy just retaining the most unstable nodal diameter. A detailed discussion of this solution is outside of the scope of this paper.

The purpose of this exercise was to prove that the proposed method provides a similar solution as the full-annulus case, both in terms of spatial and temporal harmonics in the final statistically converged state. In this operating point and with the selected level of forcing the solution is unstable but with a limited amount of unsteadiness and a relatively simple spectrum. The behavior of the different cases, with different inlet distortion screens, but with the same amplitude $\left(\triangle P_{0} \simeq 3500 P a\right)$ of the most non-linearly unstable nodal diameter are similar. It is concluded that the predictions derived from the nonlinear stability maps are valid. The three selected cases are unstable as it was predicted.

Computational Efficiency The speedup factor, $R$, of the PSM with respect a full-annulus simulation is directly related to the ratio between the number of reduced-passages samples used to approximate the simulation, and the number of blades, which in this case is 22 , since the overhead of the method is negligible. The speedup factor with respect the direct full-annulus solution using three reduced-passages samples in this case is then $R=22 / 3=7$. Taking into account that the same stall process and stability behaviour can be simulated retaining just the most unstable ND a significant speedup with respect the use of realistic more complex screens can be obtained, Table 2 compares the computational effort requires for the new methodology against a full-annulus simulation. A single full-annulus solution can be converged overnight in a cluster of 16 commodity GPUs assuming that the case is run for about 10 rotor revolutions. The nonlinear stability maps can be computed in about the same time in the same cluster.

\section{PROPOSED METHODOLOGY}

This section aims at proposing an efficient strategy that can be used to assess fan stability subject to inlet distortion quickly.

Firstly, for the selected fan blade and OP, the nonlinear stability maps equivalent to those shown in Fig. 5 should be computed. On the view of the shape of the stability boundary for all the NDs 


\begin{tabular}{|l|c|c|}
\hline & PSM & full-annulus \\
\hline \hline Number of points & $6.8 M$ & $50 M$ \\
\hline Number of GPUs (GTX 1080 Ti) & 2 & 16 \\
\hline Wall clock time per revolution (h) & 1.5 & 1 \\
\hline
\end{tabular}

TABLE 2. Computational cost of different numerical approximations for NASA rotor 67 case under distorted inflow conditions for a mesh of 2.3 M nodes per passage.

two points per nodal diameter should suffice. If just the four first NDs are computed this analysis could be conducted overnight in a modest cluster with 16 GTX 1080ti GPUs.

Secondly, the inlet distortion pattern of interest is Fourier decomposed and the stability of its first spatial harmonics assessed using the previously computed nonlinear stability maps. This process is inexpensive and the stability of a variety of inlet distortion patterns could be readily assessed.

Finally the suspicious patterns could be assessed using either a full-annulus analysis or a PSM simulation retaining more spatial harmonics.

The impact of the different airfoil shapes or loadings could be quickly assessed also if some assumptions are made about the relevant NDs. If it is concluded that just the 1st ND is relevant just the PSM simulation of this ND need to be kept in the loop.

\section{CONCLUSIONS}

Unsteady simulations with various inlet distortion patterns have been carried out for the NASA rotor 67 fan blade to investigate its aerodynamic stability under the effect of different distortion screens. The reduced order Passage Spectral Method and fullannulus representations of the fan have been used. The PSM has been slightly modified aiming at handling inlet distortion screens in a nodal diameter by nodal diameter basis efficiently without making any hypothesis about its temporal periodicity.

The proposed methodology is based on the assumption that the spatial harmonics of inlet distortion screen evolve independently, even in the nonlinear stability regime. However, the method makes no hypothesis about the interaction betwen the diferent spatial modes. In this sense the method is fully nonlinear. It is concluded that at the design operating point the mostnon-linearly unstable nodal diameter is the first which exhibits a sub-critical bifurcation.

It has been shown that three different physical inlet distortion patterns but with the same 1st ND total pressure deficit content can destabilize a stable operating point under clean inflow conditions in a similar way. Stall point for one of the distorted screen pattern has been found with full-annulus simulations to estimate the error, which is approximately $10 \%$, in the iteration procedure to build the stability map and verify the stability analysis with a full-annulus solution. Moreover, the harmonic content of the three final quasi-periodic states have been characterized concluding that all the three are very similar since they are dominated by the nonlinear instability of the 1st nodal diameter.

A simplified efficient methodology to assess the effect of intake distortion on fan stability has been proposed. The proposed methodology can produce reliable predictions of the nonlinear stability of the fan blade under the presence of complex inlet total pressure patterns without recurring to full-annulus simulations. A significant reduction in computational resources (about a factor of 7) with respect to the full-annulus analysis has been obtained for this problem.

The efficiency of the methodology is based on two cornerstones: the analysis of only the most nonlinearly unstable nodal diameter and the novel passage spectral method which does not require the use of full-annulus simulations.

\section{ACKNOWLEDGMENTS}

DR and RC wish to thank Industria de Turbopropulsores S.A.U. for allowing the publication of this paper and for its support during the project. This work has been carried-out under the RETOS project RTC1814120123 named ENVIDIA the grant from the Spanish Ministerio de Economía y Competitividad DPI201784700-R named AEROMOT.

\section{NOMENCLATURE}

$k \quad$ number of spatial harmonics

$m$ spatial harmonic index

$n \quad$ time step level

$N_{p} \quad$ number of reduced-passages samples

ND nodal diameter

$P_{0} \quad$ inlet stagnation pressure

$P_{t} \quad$ total pressure

$P R \quad$ total pressure ratio

$R \quad$ speedup factor

$S M_{L} \quad$ stall margin loss

U vector of conservation variables

$\hat{\mathbf{U}} \quad n-t h$ spatial harmonic of $\mathbf{U}$ values

$\mathbf{x} \quad$ cylindrical coordinate vector

\section{Greek Symbols}

$\theta \quad$ global circumferential coordinate $[\mathrm{rad}]$

$\omega \quad$ angular frequency $[\mathrm{rad} / \mathrm{s}]$

$\Omega_{\text {rotor }} \quad$ angular rotational speed of the rotor $[\mathrm{rad} / \mathrm{s}]$

Acronyms 
CFD Computational Fluid Dynamics

OP Operating Point

PSM Passage-Spectral Method

URANS Unsteady Reynolds-Averaged Navier-Stokes

\section{REFERENCES}

[1] Cousins, W. T., 2004. "History, philosophy, physics, and future directions of aircraft propulsion system/inlet integration". In ASME Turbo Expo 2004: Power for Land, Sea, and Air, American Society of Mechanical Engineers Digital Collection, pp. 305-320.

[2] Burstadt, P. L., Calogeras, J., and Mehalic, C., 1971. "Experimental investigation of the effect of screen induced total pressure distortion on turbojet stall margin". Technical Memorandum NASA TM X-2239, NASA Lewis Research Center, Cleveland, $O H$.

[3] Rademakers, R., Bindl, S., Niehuis, R., et al., 2016. "Effects of flow distortions as they occur in s-duct inlets on the performance and stability of a jet engine". Journal of Engineering for Gas Turbines and Power, 138(2).

[4] Page, J. H., Hield, P., and Tucker, P. G., 2018. "Effect of inlet distortion features on transonic fan rotor stall". Journal of Turbomachinery, 140(7), p. 071008.

[5] Frohnapfel, D. J., and O'Brien, W. F. "Fan response to inlet swirl distortions produced by boundary layer ingesting aircraft configurations". 51st AIAA/SAE/ASEE Joint Propulsion Conference.

[6] Hall, D. K., Greitzer, E. M., and Tan, C. S., 2017. "Analysis of fan stage conceptual design attributes for boundary layer ingestion". Journal of Turbomachinery, 139(7).

[7] Lee, K.-B., Wilson, M., and Vahdati, M., 2019. "Effects of inlet disturbances on fan stability". Journal of Engineering for Gas Turbines and Power, 141(5), p. 051014.

[8] Zhang, W., and Vahdati, M., 2018. "A parametric study of the effects of inlet distortion on fan aerodynamic stability". Journal of Turbomachinery, 141(1), p. 11 pages.

[9] Schneck, W., Ferrar, A., Bailey, J., Hoopes, K., and O'Brien, W., 2013. "Improved prediction method for the design of high-resolution total pressure distortion screens". In 51st AIAA Aerospace Sciences Meeting including the New Horizons Forum and Aerospace Exposition, p. 1133.

[10] Hah, C., Rabe, D. C., Sullivan, T. J., and Wadia, A. R., 1998. "Effects of inlet distortion on the flow field in a transonic compressor rotor". Journal of turbomachinery, 120(2), pp. 233-246.

[11] Ferrar, A., Schneck, W., Bailey, J., Hoopes, K., and O'Brien, W, 2012. "Application of additive manufacturing to rapidly produce high-resolution total pressure distortion screens". In 50th AIAA Aerospace Sciences Meeting including the New Horizons Forum and Aerospace Exposi- tion, p. 929 .

[12] Zhang, W., and Vahdati, M., 2017. "Influence of the inlet distortion on fan stall margin at different rotational speed". In GPPS conference paper, Vol. 207.

[13] Reid, C., 1969. "The response of axial flow compressors to intake flow distortion". In A.SME 1969 Gas Turbine Conference and Products Show, American Society of Mechanical Engineers, pp. V001T01A029-V001T01A029.

[14] Biesiadny, T. J., Braithwaite, W. M., Soeder, R. H., and Abdelwahab, M., 1986. "Summary of investigations of engine response to distorted inlet conditions". NASA Technical Memo No. 87317, Lewis Research Center, Cleveland, $O H$.

[15] Longley, J., Shin, H.-W., Plumley, R., Silkowski, P., Day, I., Greitzer, E., Tan, C., and Wisler, D., 1996. "Effects of rotating inlet distortion on multistage compressor stability". Journal of Turbomachinery, 118(2), pp. 181-188.

[16] Perovic, D., Hall, C. A., and Gunn, E., 2019. "Stall inception in a boundary layer ingesting fan". Joumal of Turbomachinery, 141(9), p. 091007.

[17] Yao, J., Gorrell, S. E., and Wadia, A. R., 2010. "Highfidelity numerical analysis of per-rev-type inlet distortion transfer in multistage fans - part $i$ : Simulations with selected blade rows". Journal of Turbomachinery, 132(4), p. 041014.

[18] Yao, J., Gorrell, S. E., and Wadia, A. R., 2010. "Highfidelity numerical analysis of per-rev-type inlet distortion transfer in multistage fans - part ii: Entire component simulation and investigation". Journal of Turbomachinery, $132(4)$, p. 041015.

[19] Sheoran, Y., Bouldin, B., and Krishnan, P. M., 2012. "Compressor performance and operability in swirl distortion". Jounal of turbomachinery, 134(4).

[20] Choi, M., Vahdati, M., and Imregun, M., 2011. "Effects of fan speed on rotating stall inception and recovery". Journal of Turbomachinery, 133(4), p. 041013.

[21] Choi, M., Smith, N. H., and Vahdati, M., 2013. "Validation of numerical simulation for rotating stall in a transonic fan". Journal of Turbomachinery, 135(2), p. 021004.

[22] Fidalgo, V. J., Hall, C., and Colin, Y., 2012. "A study of fan-distortion interaction within the nasa rotor 67 transonic stage". Joumal of Turbomachinery, 134(5), p. 051011.

[23] Lee, K.-B., Dodds, J., Wilson, M., and Vahdati, M., 2018. "Validation of a numerical model for predicting stalled flows in a low-speed fan-part ii: Unsteady analysis". Journal of Turbomachinery, 140(5), p. 051009.

[24] Hall, K. C., and Lorence, C. B., 1993. "Calculation of three-dimensional unsteady flows in turbomachinery using the linearized harmonic euler equations". Journal of Turbomachinery, 115(4), pp. 800-809.

[25] He, L., 2006. "Fourier modeling of steady and unsteady nonaxisymmetrical flows". Journal of propulsion and 
power, 22(1), pp. 197-201.

[26] Burgos, M. A., Contreras, J., and Corral, R., 2011. "Efficient edge-based rotor/stator interaction method". AIAA journal, 49(1), pp. 19-31.

[27] Corral, R., Gisbert, F., and Pueblas, J., 2017. "Efficient execution of a parallel edged-based navier-stokes solver on graphics processing units". Int. J. Comp. Fluid Dynamics, 31(2), pp. 1-16.

[28] Romera, D., and Corral, R., 2019. "Efficient passagespectral method for unsteady flows under stall conditions". In ASME Turbo Expo 2019, Vol. Volume 2C: Turbomachinery of Turbo Expo: Power for Land, Sea, and Air, p. V02CT41A035.

[29] Peterson, M. W., Gorrell, S. E., and List, M. G., 2017. "Fourier descriptors for improved analysis of distortion transfer and generation". In ASME Turbo Expo 2017: Turbomachinery Technical Conference and Exposition, American Society of Mechanical Engineers Digital Collection. 